\title{
Non-Surgical Endodontic Management of External Root Resorption in a Permanent Maxillary Central Incisor Tooth - A 3 Year Follow-Up of a Rare Case Scenario
}

\author{
Kothandaraman Sathyanarayanan ${ }^{1}$, Lingeswaran Poornima², Keerthi V. Narayan³
}

\author{
${ }^{1}$ Department of Conservative Dentistry and Endodontics, Sathyabama Dental College and Hospital, Chennai, \\ Tamil Nadu, India, ${ }^{2}$ Department of Conservative Dentistry and Endodontics, Teeth Care Dental Clinic, \\ Chennai, Tamil Nadu, India, ${ }^{3}$ Department of Oral Pathology and Microbiology, Thai Moogambigai Dental \\ College and Hospital, Dr M.G.R. Educational and Research Institute, Chennai, Tamil Nadu, India.
}

\section{INTRODUCTION}

Resorption of the tooth represents a multifactorial and a perplexing problem for all clinicians resulting in complete or partial loss of tooth structure. The present clinical demonstration describes management of the permanent maxillary left central incisor (tooth number \#21) affected by external root resorption involving the mesial and distal surface of middle one-third of the root. Conventional nonsurgical endodontic treatment followed by MTA (Mineral trioxide aggregate) obturation (PRoRoot MTA, Dentsply, Tulsa Dental Specialties) was planned. The root canal of the affected teeth was debrided with Dual Rinse HEDP (Medcem Weinfelden, Switzerland) containing etidronate powder and chemomechanical preparation of the root canal was performed with XP endo shaper file system (FKG Dentaire, SA, Switzerland). Thirtysix months' post-operative follow-up revealed complete healing of the external root resorption defect with no clinical and radiological signs and symptoms. In the present case simple non-surgical endodontic management of severe external root resorption was done in a permanent maxillary tooth by using a continuous chelation irrigation technique, intracanal medicament followed by obturation with bio-ceramic material produced satisfactory results in contrast to the recommended surgical management.

Resorption of the tooth being a multifactorial and a perplexing problem for all clinicians results in complete or partial loss of tooth structure. According to the Glossary of Endodontic terms, Resorption is defined as a condition associated with either a physiologic or a pathologic process resulting in the loss of dentine, cementum, and/or bone. ${ }^{1}$ Traditionally resorption can present either as internal or as external resorption. ${ }^{2}$ External root resorption occurs on the outer surface of the root and are of dissimilar nature such as external surface resorption, external inflammatory root resorption, ankylosis, and external replacement resorption, the most common being external inflammatory root resorption. ${ }^{3}$ According to the Glossary of Endodontics, "Inflammatory resorption is defined as an internal or external pathologic loss of tooth structure and possibly bone, resulting in a defect; occurs as the result of microbial infection; characterized radiographically by radiolucent areas along the root". ${ }^{1}$ It may result due to dental trauma, forceful orthodontic tooth movement, long standing infection of the pulp or periodontal tissues. External resorption presents as a major resorptive condition of the root without any clinical signs and symptoms. ${ }^{2}$ The non-surgical management of external inflammatory root resorption is based on its aetiology, which needs to be eliminated. ${ }^{3}$
Corresponding Author:

Dr. Sathyanarayanan Kothandaraman Reader, Department of Conservative Dentistry and Endodontics, Sathyabama Dental College and Hospital, Chennai - 600119,

Tamil Nadu, India.

E-mail: drsathya_ods@yahoo.com

DOI: $10.14260 / j e m d s / 2021 / 481$

How to Cite This Article: Sathyanarayanan K, Poornima L, Narayan $K V$, et al. Non-surgical endodontic management of external root resorption in a permanent maxillary central incisor tooth - a 3-year follow-up of a rare case scenario. J Evolution Med Dent Sci 2021;10(30): 2350-2353, DOI:

$10.14260 / \mathrm{jemds} / 2021 / 481$

Submission 16-05-2021,

Peer Review 01-07-2021

Acceptance 11-07-2021,

Published 26-07-2021.

Copyright (C) 2021 Kothandaraman Sathyanarayanan et al. This is an open access article distributed under Creative Commons Attribution License [Attribution 4.0 International (CC BY 4.0)] 
The main objective of endodontic management is the removal of necrotic pulpal remnants from the root canal system and to reduce the bacterial load below the tolerable level. Calcium hydroxide is the choice of intracanal medicament for the treatment of external inflammatory root resorption. ${ }^{4}$ Over the years several studies have shown that longer treatment with calcium hydroxide is beneficial when there has been a delay in the removal of necrotic pulpal remnants. ${ }^{5}$ Root canal irrigants most commonly used for necrotic pulp tissue dissolution, killing the microorganisms and smear layer removal are sodium hypochlorite $(\mathrm{NaOCl})$ followed by ethylenediamine tetra acetic acid (EDTA). ${ }^{6}$ However, EDTA when combined with $\mathrm{NaOCl}$ results in chemical interaction and exothermic reaction consuming free available chlorine in $\mathrm{NaOCl}$ solutions, thus reducing its tissue dissolving and microbicidal properties. ${ }^{7}$ Further, EDTA will result in complete demineralization and erosion of exposed peritubular dentin. ${ }^{8}$ To overcome the above disadvantages, the concept of continuous chelation protocol was introduced by mixing $\mathrm{NaOCl}$ with a less aggressive chelator. $7 \%$ to $10 \%$ of 1 - hydroxyethylidene - 1, 1 - bisphosphonate (HEBP) can be used during root canal preparation without any loss of $\mathrm{NaOCl}$ efficacy. ${ }^{9,10}$ Mineral trioxide aggregate (MTA) is a hydrophilic material and sets in the presence of water. MTA known for its biocompatibility has been used as a retro filling material and to seal root perforations. ${ }^{10,11}$

Thus, it has an ability to regenerate peri radicular tissues, such as bone and cementum, and can be considered as a viable option for root canal filling in external root resorption after delayed tooth replantation. ${ }^{12}$ This clinical case report describes the endodontic management of external root resorption with white MTA as an obturating material. This case is unique as its presentation in a permanent tooth root resorption is uncommon owing to the inert antiresorptive properties of the precementum covering the root. External root resorption has been managed by using continuous chelation irrigation with dual rinse HEDP followed by obturation with white - MTA at 36 months follow up.

\section{PRESENTATION OF CASE}

A 24-year-old Indian male patient was referred to the Department of Conservative Dentistry and Endodontics (Sathyabama Dental College and Hospital, Chennai, Tamilnadu, India) for the management of his maxillary right (\#11) and left central incisors (\#21) respectively. The patient had a traumatic history due to impact injury of his upper front teeth. Patient's past medical history was non - contributory. On clinical examination there was no mobility of tooth \#11 but there was grade 2 mobility of tooth \#21. Clinically, the gingival attachment was normal and the palpation of the labial and palatal aspect of the \#11 \& \#21 revealed no tenderness. Nevertheless, \#11 and \#21 exhibited tenderness to percussion. Periodontal probing depth was within physiological limits around \#11 and \#21. Pulp sensibility tests revealed a negative response. The pre-treatment radiograph of \#21 had a resorbed bowl - shaped defect on the external surface of middle one-third of the root. The root resorption affected the outer aspects of the mesial and distal surface of middle one-third of the maxillary left central incisor. Periapical radiolucencies were seen in both \#21 \&\#11 (Fig. 1).

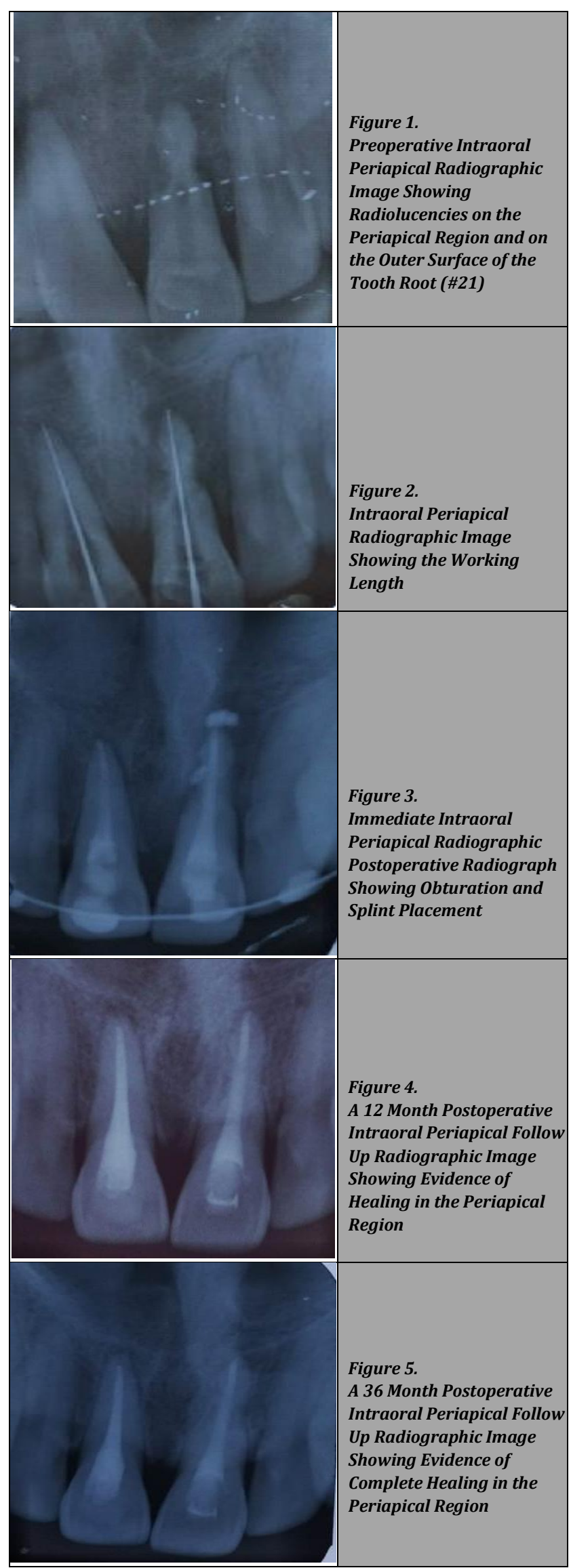




\section{PROVISIONAL DIAGNOSIS}

On clinical and radiographic findings, \#11 was diagnosed as pulp necrosis with symptomatic apical periodontitis and \#21 as external inflammatory root resorption with symptomatic apical periodontitis.

\section{DISCUSSION OF MANAGEMENT}

\section{Treatment Planning}

Conventional nonsurgical endodontic management with inter appointment calcium hydroxide as intra-canal medicament was the preferred treatment of choice for external inflammatory root resorption with symptomatic apical periodontitis.

\section{Procedure}

\section{Visit 1}

During the first visit, local anaesthesia was administered with $2 \%$ lidocaine with 1:200,000 epinephrine (Xylocaine; AstraZeneca Pharma India Ltd, Bengaluru, India), under rubber dam isolation, endodontic access cavity was prepared for \#11 \& \#21 with an endo-access bur and an endo Z bur (Dentsply Maillefer, Tulsa Dental, USA). Working lengths were determined with \#20 file (Mani Inc., Utsunomiya, Japan) with the help of an electronic apex locator (Root ZX; J. Morita, Tokyo, Japan) and verified with radiographs (Figure 2). Chemo mechanical procedure of the canal was performed using \#30/.04 XP - endoshaper file system (XP; FKG Dentaire, La Chaux - deFonds, Switzerland) and debridement was carried out by continuous chelation irrigation technique with $2.5 \%$ $\mathrm{NaOCl}$ combined with $9 \%$ HEDP which is commercially available as Dual Rinse HEDP (Medcem Weinfelden, Switzerland). An irrigation facilitator - EndoActivator System (Dentsply Sirona, Ballaigues, Switzerland) was included for thorough cleaning of the root canal system. Calcium hydroxide (RC Cal, Prime Dental, Bhiwandi, India) was placed in the root canal as an inter-appointment intracanal medicament and the access cavity was sealed with Cavit G (3M ESPE, St. Paul, MN, USA). Splinting of the maxillary anterior was done to reduce the grade 2 mobility of tooth \#21. The next appointment was scheduled for two weeks.

\section{Visit 2}

In the second visit, teeth were isolated with rubber dam and intracanal medicament was removed from the canals by thorough irrigation with saline, the root canals of \#11 \& \#21 were dried with absorbent paper points (Dentsply Maillefer, Tulsa, USA). Master cone radiograph was taken for \#11 and obturated with gutta-percha points of $2 \%$ taper and MTA based root canal sealer (MTA Fillapex, Angelus, Londrina, Brazil) by cold lateral compaction technique. The root canal of tooth \#21 was obturated with white MTA (ProRoot MTA, Dentsply, Tulsa Dental) as an obturating material (Figure 3). After completion of endodontic procedure, the access cavities of \#11 \& \#21 were restored with a composite resin (Te Econom Plus, Ivoclar Vivadent, India).

\section{Post-Treatment Follow-Up}

Patient was reviewed over a period of thirty - six months both clinically and radiographically. Postoperative radiographs (Figure 4,5) revealed complete healing of the external root resorption defect in \#21 with no clinical and radiographical postoperative signs or symptoms of failure.

\section{DISCUSSION}

Successful management of external root resorption in trauma patients is of utmost importance for a clinician to achieve favourable healing which varies from one person to another owing to several local and systemic factors. ${ }^{13}$ Trauma and pulp space infection predominantly contributes to external root resorption. ${ }^{14}$ When a tooth sustains a traumatic injury, it leads to varying degrees of attachment loss. The cemental covering of the root is damaged and its protective quality is lost. If the root canal is infected, the bacterial toxins can penetrate through the dentinal tubules and exert damage to periodontal ligament, which results in the resorption of root.14,15 Inflammatory resorption is a mechanism by which infected tissues are removed. ${ }^{16}$ Osteoclasts being a specialized macrophages aids in the healing process of traumatized teeth and bone. Osteoclasts tend to remove infected calcified tissues, and this progress is done till the aetiology is removed. ${ }^{14}$

Non-surgical endodontic therapy with the use of continuous chelation Dual Rinse HEDP, a bisphosphonate root canal irrigant followed by an intracanal medicament and MTA obturation was often the recommended treatment of choice in such cases. In the continuous chelation concept, $\mathrm{NaOCl}$ is mixed with soft chelators such as etidronate powder, a nitrogen - free bisphosphonates appear to have a minimal effect on dentin walls but remove smear layer effectively. ${ }^{17,18}$ This combination stabilizes the hypochlorite concentration, which results in enhanced tissue dissolution and is found to be less cytotoxic. ${ }^{19,20} \mathrm{NaOCl}$ - Dual Rinse HEDP irrigating solution exhibited better haemostasis when compared to EDTA in case of root resorption, perforation, and maintenance of bleaching effect of $\mathrm{NaOCl}$ in blood stained dentin. When HEDP is mixed with $\mathrm{NaOCl}$, the proteolytic effect of $\mathrm{NaOCl}$ predominates with reduced bleeding when compared to EDTA. ${ }^{21}$ Etidronates belong to bisphosphonates, and they inhibit osteoclastic activity and exhibit antiinflammatory properties. Etidronate salts when mixed with $\mathrm{NaOCl}$ increases its surface tension and may reduce the wettability of the combined irrigant to the root canal dentinal walls. ${ }^{22}$ Hence, an endo activator system with safe, noncutting polymer tips to vigorously agitate irrigant solution was used.

The calcium hydroxide dressing helps to control infection and maintains the root canal with alkaline $\mathrm{pH}$, an environment conducive for calcified tissue formation. Calcium hydroxide due to its high calcium ion concentration and alkaline $\mathrm{pH}$ is used for the treatment of external root resorption. ${ }^{4}$ There are various theories for its mechanism of action like stimulating the formative cells and neutralizing the action of resorptive cells by its high $\mathrm{pH} .23$

After thorough debridement, cleaning and shaping was done with XP endo shaper file system. The advantage of this system is that it minimizes the stresses exerted into root canal dentinal walls and prevents the risk of micro cracks. It has a gentle, non - aggressive action and promotes conservative root 
canal preparation. Further, it has a smaller core than conventional instruments which facilitates debris removal without occluding the dentinal tubules. ${ }^{24}$ MTA, due to its alkaline $\mathrm{pH}$ releases calcium ions even after its setting reaction.11,12 These calcium ions penetrate through the dentinal defects in root canals restored with MTA, and the concentration increases with time. ${ }^{25}$ In this clinical case, the calcium hydroxide dressing and MTA obturation had a synergistic effect and helped in the healing process. However external root resorption defect can be accurately evaluated by Cone Beam Computed Tomography (CBCT). Due to radiation exposure to the patient, it was not considered in this clinical report.

\section{CONCLUSIONS}

In this clinical presentation non-surgical endodontic treatment combined with calcium hydroxide intracanal medicament and MTA obturation was performed for the management of external root resorption. Further, continuous chelation with Dual Rinse HEDP irrigating solution and endo activator system helped in thorough cleaning of the root canal that ensured a better prognosis and healing of the external root resorption defect. Thus, meticulous case selection by appropriate examination and prompt diagnosis combined with treatment promoting complete inactivation of resorptive tissue and regular follow-up, can aid in successful management.

Financial or other competing interests: None.

Disclosure forms provided by the authors are available with the full text of this article at jemds.com.

\section{REFERENCES}

[1] American Association of Endodontists. Glossary of endodontic terms. $10^{\text {th }}$ edn. American Association of Endodontists 2020.

[2] Andreasen J0. Luxation of permanent teeth due to trauma. A clinical and radiographic follow-up study of 189 injured teeth. Scand J Dent Res 1970;78(3):273-86.

[3] Gunraj MN. Dental root resorption. Oral Surg Oral Med Oral Pathol Oral Radiol Endod 1999;88(6):647-54.

[4] Saad AY. Calcium hydroxide in treatment of external root resorption. J Am Dent Assoc 1989;118(5):579-81.

[5] Trope M, Moshonov J, Nissan R, et al. Short vs. long-term calcium hydroxide treatment of established inflammatory root resorption in replanted dog teeth. Endod Dent Traumatol 1995;11(3):124-8.

[6] Zehnder M. Root canal irrigants. J Endod 2006;32(5):38998.

[7] Grawehr M, Sener B, Waltimo T, et al. Interactions of ethylenediamine tetra acetic acid with sodium hypochlorite in aqueous solutions. Int Endod J 2003;36(6):411-7.
[8] Wang Z, Maezono H, Shen Y, et al. Evaluation of root canal dentin erosion after different irrigation methods using energy-dispersive X-ray spectroscopy. J Endod 2016;42(12):1834-9.

[9] Zehnder M, Schmidlin P, Sener B, et al. Chelation in root canal therapy reconsidered. J Endod 2005;31(11):817-20.

[10] Parirokh M, Torabinejad M. Mineral trioxide aggregate: a comprehensive literature review--part III: clinical applications, drawbacks and mechanism of action. J Endod 2010;36(3):400-13.

[11] Camilleri J, Ford TRP. Mineral trioxide aggregate: a review of the constituents and biological properties of the material. Int Endod J 2006;39(10):747-54.

[12] Marão HF, Panzarini SR, Aranega AM, et al. Periapical tissue reactions to calcium hydroxide and MTA after external root resorption as a sequela of delayed tooth replantation. Dent Traumatol 2012;28(4):306-13.

[13] Heithersay GS. Management of tooth resorption. Aust Dent J 2007;52(1 Suppl):S105-21.

[14] Trope M. Root resorption due to dental trauma. Endodontic Topics 2002;1(1):79-100.

[15] Abbott PV. Prevention and management of external inflammatory resorption following trauma to teeth. Aust Dent J 2016;61(Suppl 1):82-94.

[16] Andreasen JO. External root resorption: its implication in dental traumatology, pedodontics, periodontics, orthodontics and endodontics. Int Endod J 1985;18(2):109-18.

[17] Zehnder M. Etidronate (Dual Rinse HEDP) for root canal irrigation in clinical use. Quintessenz Zahnmed 2019;70:896-905.

[18] Lottanti S, Gautschi H, Sener B, et al. Effects of ethylenediaminetetraacetic, etidronic and peracetic acid irrigation on human root dentine and the smear layer. Int Endod J 2009;42(4):335-43.

[19] Christensen C, Mcneal S, Eleazer P. Effect of lowering the $\mathrm{pH}$ of sodium hypochlorite on dissolving tissue in vitro. J Endod 2008;34(4):449-52.

[20] Aubut V, Pommel L, Verhille B, et al. Biological properties of a neutralized $2.5 \%$ sodium hypochlorite solution. Oral Surg Oral Med Oral Pathol Oral Radiol Endod 2010;109(2):e120-5.

[21] Zollinger A, Attin T, Mohn D, et al. Effects of endodontic irrigants on blood and blood-stained dentin. Heliyon 2019;5(5):e01794.

[22] Giardino L, Del Fabbro M, Morra M, et al. Dual Rinse $\AA$ HEDP increases the surface tension of $\mathrm{NaOCl}$ but may increase its dentin disinfection efficacy. Odontology 2019;107(4):521-9.

[23] Mohammadi Z, Dummer PM. Properties and applications of calcium hydroxide in endodontics and dental traumatology. Int Endod J 2011;44(8):697-730.

[24] Dentaire FKG. Root canal preparations industry data. XP Endo Shaper 2016.

[25] Tronstad L, Andreasen JO, Hasselgren G, et al. pH changes in dental tissues after root canal filling with calcium hydroxide. J Endod 1981;7(1):17-21. 